\title{
Expression of Xyloglucanase (AaXEG2) in Open-field-grown Poplars
}

\author{
Haruka lizuka, Tomomi Kaku, Toru Taniguchi, Manabu Kurita, Ken-ichi Konagaya, Katsuaki Ishii, \\ Teiji Kondo, Kei'ichi Baba, Rumi Kaida, Teruaki Taji, Yoichi Sakata, and Takahisa Hayashi
}

\begin{abstract}
The transgenic expression of Aspergillus aculeatus xyloglucanase with $35 \mathrm{~S}$ promoter, which has been maintained in greenhouse-grown transgenic poplars for 10 years, was assessed to determine their expression level in open-field-grown poplars. This level was slightly decreased in the poplars grown in fertile soil for four years, and there were proportionately decreased levels of xyloglucanase activity among their tissues, in which the shoots exhibited slightly lower specific activities than was observed in the leaves and roots. Xyloglucan content was greatly decreased in the walls of the tissues in the transgenic lines, although the degradation levels of xyloglucan were slightly lower for the poplars grown in fertile soil than for those grown in non-fertile soil. The results showed that the level of expression, together with xyloglucan degradation in their walls, was decreased for the poplars grown in fertile soil.
\end{abstract}

Keywords: xyloglucan, xyloglucanase, field trial, poplar, transgenic expression, soil nutrition.

\section{Introduction}

Since adjacent cellulose microfibrils are thought to be cross-linked by xyloglucans, the separation of microfibrils during elongation has been thought to require enzymes that cleave xyloglucan or loosen its binding to microfibrils (Hayashi 1989a). Involvement of the cleavage of xyloglucan in cell elongation also agrees with a previous finding (Takeda et al. 2002) that the integration of xyloglucan oligosaccharides into pea stem segments solubilizes endogenous xyloglucan in the wall, weakens the cell wall, and accelerates cell elongation. The decreased amount of tethering xyloglucan was attributable to a decreased cross-linking component, which could in turn accelerate growth by the increased plastic extensibility under turgor pressure.

Aspergillus xyloglucanase cDNA (AaXEG) with $35 S$ promoter has been used for the expression studies in open-field-grown poplars because the overexpression of xyloglucanase could affect wall dynamics (Park et al. 2004). Genomic Southern hybridization showed that the transgene might exist as one copy for trg300-1 and two copies for trg300-2 at different loci (Taniguchi et al. 2008). The genes, which are integrated in genomes at a low copy number, as was true for these transgenic poplars, are expected to be expressed stably. Six plots of poplar plantations were used for the field trial, of which three plots contained fertile soil and three contained non-fertile soil. Both of these three-plot sets consisted of one plot each of trg300-1, trg300-2 and wild type plants, and each individual plot of $144 \mathrm{~m}^{2}$ contained 25 trees arranged in a $5 \times 5$ grid with $2 \times 2 \mathrm{~m}$ spacing between the plants. The growth level of the plants in each plot will be shown in other papers. This paper describes the levels of xyloglucanase activities among the different tissues, together with xyloglucan degradation in the walls of the open-field-grown transgenic poplars.
The level of transgenic expression for xyloglucanase was assessed in this study since earlier studies have shown some transgene instability in transgenic plants, where gene silencing might occur due to genome rearrangement, DNA methylation, insertion site interaction, etc (Flavell 1994; Ulian et al. 1996; Elmayan and Vaucheret 1996; Palauqui and Vaucheret 1998). Since trees have a long life span, some genetic regulation could occur in mature tissues due to different stresses such as strong winds, insect attacks, excessive water during rainy season, drought, typhoon, earthquake, and/or high and low temperatures. There was at least one typhoon and one powerful earthquake which hit this field from March 23, 2007 to December 14, 2010. Two transgenic lines, trg300-1 and $\operatorname{trg} 300-2$, were produced in March 2000 and the level of transgenic expression was stable until March 2007 under greenhouse conditions. The level of the expression and xyloglucan content could change due to environmental factors affecting the poplars during their four years in the field. This paper assesses whether transgenic traits could remain stable for open-field-grown transgenic poplars.

\section{Materials and Methods}

\section{Plant Materials}

Wild type and two transgenic-line poplars (trg300-1 and trg300-2) were used in this experiment. Shoots, leaves, xylems and roots were harvested from each line in July 2010 .

\section{Soil Conditions}

Two kinds of soil were used for the field trials of the transgenic poplars. One was simply a forest-field soil, which contained average levels of soil nutrients found in Japanese forests. The other was a farming-field soil, which contained average levels of soil nutrients found in Japanese farming fields. The former was defined as a 
fertile soil and the latter as a non-fertile soil. Both types were layered onto the experimental field which was located in the Forest Breeding Center in Japan (http://ftbc.job.affrc.go.jp/).

\section{Xyloglucanase Analysis}

Each tissue was homogenized with liquid nitrogen, and the resulting powder was ground with $20 \mathrm{mM}$ sodium phosphate buffer ( $\mathrm{pH} 6.2$ ) containing $1 \mathrm{M} \mathrm{NaCl}$ in a mortar and the wall residue was washed three times. The combined supernatant was adjusted to $80 \%$ saturation with solid ammonium sulfate to generate a precipitate from which an aliquot protein was used. Differentiating xylem was scraped with a knife blade from differentiating tissue in which the bark had been peeled off (Baba and Ito 1997). The xylem was immediately put into liquid nitrogen and ground to a fine powder with liquid nitrogen in a mortar. The resulting fine powder was extracted with $10 \mathrm{ml}$ of methanol containing $0.1 \%$ dithiothreitol at room temperature. The mixture was centrifuged to remove its supernatant. This extraction procedure was repeated three times and the precipitate was dried under reduced pressure. The resulting powder was then ground with $20 \mathrm{mM}$ sodium phosphate buffer ( $\mathrm{pH}$ 6.2) containing $1 \mathrm{M} \mathrm{NaCl}$ in a mortar as described above.

Proteins $(2 \mathrm{gg})$ were subjected to electrophoresis with $10 \%$ sodium dodecyl sulfate-polyacrylamide gel electrophoresis (SDS-PAGE) and electrotransferred to Hybond-C Extra (Amersham, Uppsala, Sweden), and probed with an antibody against AaXEG2, followed by a second antibody using a Toyobo ABC High-HRP immunostaining kit.

Xyloglucanase activity was assayed viscometrically at $28^{\circ} \mathrm{C}$ for $2 \mathrm{~h}$ with $0.1 \mathrm{ml}$ of enzyme preparation plus $0.9 \mathrm{ml}$ of $10 \mathrm{mM}$ sodium phosphate buffer (pH 6.2) containing $1.5 \%(\mathrm{w} / \mathrm{v})$ tamarind xyloglucan in Cannon semimicroviscometers. One unit of activity is defined as the amount of enzyme required to cause $0.1 \%$ loss in viscosity in $1 \mathrm{~min}$ under such conditions.

\section{Wall Analysis}

Each tissue was homogenized with liquid nitrogen, and the resulting powder was ground with $20 \mathrm{mM}$ sodium phosphate buffer ( $\mathrm{pH}$ 6.2) containing $1 \mathrm{M} \mathrm{NaCl}$ in a mortar and the wall residue was washed three times. Each wall residue was washed with water once and successively extracted four times with $24 \% \mathrm{KOH}$ containing $0.1 \%$ $\mathrm{NaBH}_{4}$. The alkali-soluble fraction was neutralized, dialyzed with Spectra/Por dialysis membrane MWCO: 1,000 (Spectrum, Rancho Dominguez, USA), and freezedried for use in methylation analysis. Partially methylated alditol acetates were analyzed using an Agilent gas chromatography-mass spectrometer apparatus (Santa Clara, CA, USA) with a glass capillary column DB-225, (0.25 mm i.d. x15 m; Agilent). Each alditol acetate was identified by its retention time and mass spectrum (Hayashi 1989b). Xyloglucan content was calculated as the 2.86 -fold amount of 4,6-linked glucose in the alkali-soluble fraction due to the structure of xyloglucan in poplars (Hayashi and Takeda 1994). The alkali-insoluble wall residue (cellulose fraction) was washed twice with water and successively extracted with acetic/nitric reagent $(80 \%$ acetic acid/ concentrated nitric acid, 10:1) in a boiling water bath for 30 min (Updergraff 1969). The resulting insoluble material was washed in water, freeze-dried, and weighed in order to determine the amount of cellulose present.

\section{Results and Discussion}

\section{Open-field Conditions}

The open-field-grown transgenic poplars experienced a high temperature of above $36.0^{\circ} \mathrm{C}$ in August 2009 but typically experienced a high of around 30 to $35^{\circ} \mathrm{C}$ in July and August. They experienced a low of below $-7.0^{\circ} \mathrm{C}$ in February 2009 but typically a low of -6.0 to $-7.0^{\circ} \mathrm{C}$ with about $5 \mathrm{~cm}$ snowfall during December to February. Dry season occurred from November to January and rainy season from June to July. Wind speed was rather high, with an average of $15 \mathrm{~m} / \mathrm{s}$ from January to April, in which there was a maximum wind gust of $30.3 \mathrm{~m} / \mathrm{s}$ on February 2008. A $965 \mathrm{hPa}$ typhoon struck the poplars with a maximum wind gust of $50 \mathrm{~m} / \mathrm{s}$ on September 7, 2007. Earthquakes occurred numerous times in the field, twice at a magnitude of 4, 18 times at a magnitude of 3,59 times at a magnitude of 2, and 155 times at a magnitude of 1 on the Japanese seismic intensity scale. Every year, insects would attack the leaves of the poplars, during which time several plants would lose more than $25 \%$ of their leaves. It should be noted that the transgenic poplars were grown under natural and not artificial conditions.

\section{Level of Expression for AaXEG2 Gene Product (Xyloglucanase)}

Western blot analysis showed the expression of xyloglucanase (AaXEG2) in the leaf, the differentiating xylem and the root of transgenic poplars, trg300-1 and trg300-2, but not in those of the wild type plants (Figure 1). They were relatively weaker in transgenic lines grown in the fertile soil than in those in both the non-fertile soil and the greenhouse. When xyloglucanase activities were determined viscometrically in their shoots, leaves and roots, all the enzyme preparations from transgenic lines showed a drastic decrease in the viscosity of tamarind xyloglucan in a very short time period (Figure 2). Since expressed xyloglucanase exhibited very strong activity leading to xyloglucan degradation, the preparations were diluted at different concentrations.

When the activities were determined as specific activities per proteins, the transgenic poplars grown in the fertile soil showed more decreased activities than did those grown in the non-fertile soil or in the greenhouse (Table 1). It is likely that the expression level of xyloglucanase varied 
between the two kinds of soils, although they were expressed under the control of a constitutive $35 \mathrm{~S}$ promoter. Nevertheless, the specific activity was always high in the leaves and roots, but low in the shoots. It should be noted that the shoots rather than the differentiating xylem were used for the determination of xyloglucanase activity because it was difficult to obtain enzyme preparations due to the ongoing enzymic activity in the xylem (Figures 1 and 2, Table1).

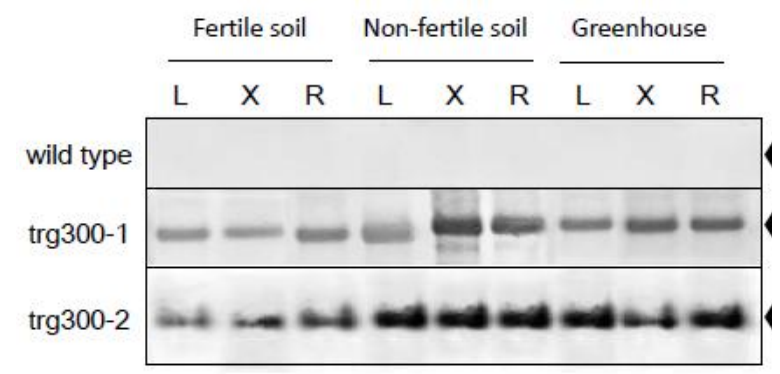

Figure 1. Western blot analysis of xyloglucanase from transgenic poplars. L, leaf; $\mathrm{X}$, differentiating xylem; $\mathrm{R}$, root. Arrows indicate a $28-\mathrm{kDa}$ gene product.

\section{Level of Xyloglucan and Cellulose in the Walls}

Xyloglucan was found at decreased levels in the tissues of the transgenic lines due to the level of 4,6-linked glucose, by which the polysaccharide in xylem became even less than that in the shoots, leaves and roots (Table 2). The levels of xyloglucan were always higher in the shoots (22.0 to $22.2 \%$ of xyloglucan compared with that in the wild type poplars), leaves (21.1 to $22.2 \%$ ), xylem (17.5 to $18.3 \%$ ) and roots (18.3 to $19.2 \%$ ) of the transgenic lines grown in the fertile soil than those in the shoots $(17.9$ to $21.3 \%$ ), leaves (16.3 to $16.9 \%$ ), xylem (14.3 to $15.0 \%$ ) and roots $(16.4$ to $17.3 \%)$ of the lines grown in the non-fertile soil. The levels of the transgenic lines grown in the nonfertile soil and in the greenhouse were very similar. Therefore, the degradation levels were slightly more pronounced in the poplars grown in the fertile soil.
Cellulose content was increased in all the walls of the transgenic lines, in which the increased level of cellulose was slightly higher in the xylem. The level was also slightly decreased to $105 \sim 107 \%$ in the xylem of the lines in December 2010 from 109 111\% in December 2002 (Park et al. 2004). To date, no significant differences have been observed in cellulose content between poplars grown in fertile soil and in non-fertile soil.

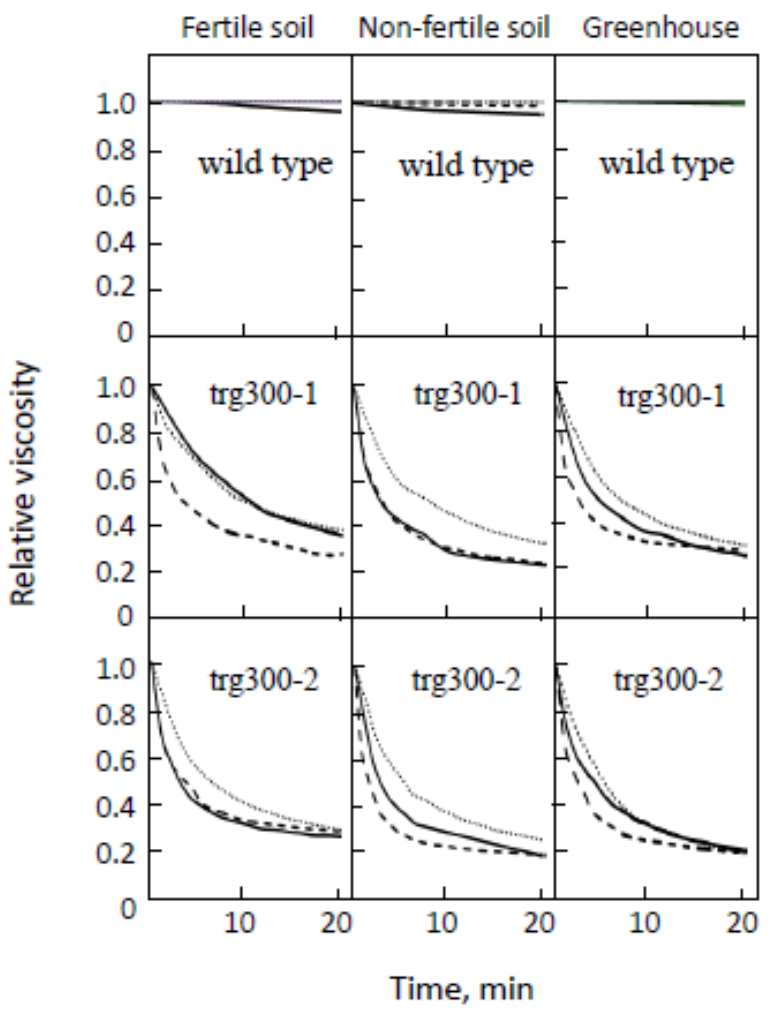

Figure 2.Time course of xyloglucan degradation. Dotted lines (......) indicate shoots, dashed lines ( leaves, and straight lines (_ $)$ roots. The enzyme preparations used ranged from 0.3 to 2.6 $\mu \mathrm{g}$ of protein in the reaction mixtures.

Table 1. Specific activity of xyloglucanase.

\begin{tabular}{clccc}
\hline \multirow{2}{*}{ Plant } & Tissue & \multicolumn{3}{c}{ Specific activity ( $\times 10^{2}$ unit/mg protein) } \\
& Shoot & $0.07 \pm 0.02$ & $0.04 \pm 0.03$ & $0.05 \pm 0.02$ \\
Wild type & Leaf & $0.06 \pm 0.04$ & $0.05 \pm 0.04$ & $0.08 \pm 0.03$ \\
& Root & $0.09 \pm 0.06$ & $0.07 \pm 0.05$ & $0.08 \pm 0.03$ \\
Trg300-1 & Shoot & $2.24 \pm 0.25$ & $5.48 \pm 0.35$ & $5.19 \pm 0.45$ \\
& Leaf & $3.27 \pm 0.31$ & $7.55 \pm 0.46$ & $7.04 \pm 0.44$ \\
& Root & $3.11 \pm 0.24$ & $7.50 \pm 0.55$ & $7.00 \pm 0.56$ \\
Trg300-2 & Shoot & $2.30 \pm 0.23$ & $5.80 \pm 0.34$ & $4.68 \pm 0.37$ \\
& Leaf & $2.58 \pm 0.31$ & $7.35 \pm 0.55$ & $7.57 \pm 0.50$ \\
& Root & $3.00 \pm 0.34$ & $7.60 \pm 0.61$ & $7.30 \pm 0.56$ \\
\hline
\end{tabular}

Data represent the means \pm SE of independent plants of a single line. Values with different letters indicate statiscally significant differences according to student's t-test $(P<0.05)$. 
Table 2. Amounts of xyloglucan and cellulose in the tissues.

\begin{tabular}{|c|c|c|c|c|c|c|c|}
\hline \multirow{3}{*}{ Plant } & \multirow{3}{*}{ Tissue } & \multicolumn{6}{|c|}{ Content (mg/100 mg dry weight) } \\
\hline & & \multicolumn{2}{|c|}{ Fertile soil } & \multicolumn{2}{|c|}{ Non-fertile soil } & \multicolumn{2}{|c|}{ Greenhouse } \\
\hline & & xyloglucan & cellulose & xyloglucan & cellulose & xyloglucan & cellulose \\
\hline \multirow[t]{4}{*}{ Wild type } & Shoot & 4.10 & 30.3 & 3.90 & 31.3 & 4.10 & 31.2 \\
\hline & Leaf & 1.80 & 30.2 & 1.60 & 31.5 & 1.70 & 31.0 \\
\hline & Xylem & 0.12 & 42.5 & 0.14 & 42.7 & 0.13 & 42.8 \\
\hline & Root & 1.20 & 37.0 & 1.10 & 35.0 & 1.00 & 36.0 \\
\hline \multirow[t]{4}{*}{$\operatorname{Trg} 300-1$} & Shoot & 0.90 & 32.5 & 0.83 & 32.1 & 0.80 & 32.5 \\
\hline & Leaf & 0.40 & 33.9 & 0.27 & 32.3 & 0.29 & 34.9 \\
\hline & Xylem & 0.02 & 44.9 & 0.02 & 45.0 & 0.02 & 45.9 \\
\hline & Root & 0.23 & 37.5 & 0.19 & 36.0 & 0.18 & 36.4 \\
\hline \multirow[t]{4}{*}{$\operatorname{Trg} 300-2$} & Shoot & 0.91 & 31.7 & 0.70 & 32.5 & 0.75 & 32.4 \\
\hline & Leaf & 0.38 & 33.4 & 0.26 & 34.2 & 0.33 & 32.7 \\
\hline & Xylem & 0.02 & 45.3 & 0.02 & 45.8 & 0.02 & 46.0 \\
\hline & Root & 0.22 & 38.5 & 0.18 & 38.0 & 0.17 & 37.5 \\
\hline
\end{tabular}

Each data point represents the mean of three different experiments, with individual values varying from the mean by $<5.5 \%$.

The transgenic expression of xyloglucanase with $35 \mathrm{~S}$ promoter has caused a decrease in xyloglucan content in poplars grown using transplantations of cuttings for seven years leading up to the field trial. The expression in the transgenic lines seems to be stable in the non-fertile soil but rather unstable in the fertile soil. Although a high level of the expression and a subsequent decrease in xyloglucan content occurred in the poplars (Figure 2), the decreased level of xyloglucan might also be gradually changed for those grown in the fertile soil (Table 2). Studies on this type of expression lasting longer than four years are needed for long-living plants such as trees.

Since the high levels of xyloglucanase activity (Figures 1 and 2, Table 1) have been expressed in the walls of transgenic poplars, the residual quantity of wall-bound xyloglucan represents the xyloglucan polymer that might be tightly adhering to the microfibrils, perhaps as a monolayer coating the surfaces (Hayashi et al. 1994). However, there is evidence (Yamamoto et al. 2011) that xyloglucan does not exist in the surface of the elementary fibers in the transgenic lines. This shows that most of the residual xyloglucans in the transgenic lines occur either in the surface of microfibrils or between the elementary fibers without forming hydrogen bondings to cellulose.

This overexpression of xyloglucanase seems to be a form of xyloglucan knockout in the walls, although the gene knockout mutant of xyloglucan xylosyltransferase should cause xyloglucan knockout (Zabotina et al. 2008; Cavalier et al. 2008). However, so far, complete xyloglucan knockout has not been carried out either by the overexpression or by the gene knockout. The former may experience difficulty due to reduced accessibility of xyloglucan embedded in cellulose microfibrils for the overexpressed enzyme and the latter in the involvement of several isoenzyme genes for one transferase.

\section{Acknowledgements}

This work was supported by JSPS KAKENFIGUREHI [Grant-in-Aid for Scientific Research (A) 19208016]. This paper is also a part of the outcome of the JSPS Global COE Program (E-04): In Search of Sustainable Humanosphere in Asia and Africa.

\section{References}

Baba, K.; K. Ito. 1997. Improvement on Preparation of RNA from Differentiating Xylem Tissue of Eucalyptus camaldulensis L. Wood Research 84: 7-11.

Cavalier, D.M.; O. Lerouxel; L. Neumetzler; K. Yamaguchi; A. Reinecke; G. Freshour; O.A. Zabotina; M.G. Hahn; I. Burgert; M. Pauly; N.V. Raiknel; K. Keegstra. 2008. Disrupting Two Arabidopsis thaliana Xylosyltransferase Genes Results in Plants Deficient in Xyloglucan, A Major Primary Cell Wall Component. Plant Cell 20: 1519-1537.

Elmayan, T.; H. Vaucheret. 1996. A strongly-expressed 35Sdriven Transgene Undergoes Post-transcriptional Silencing in All Tobacco Transformants Irrespective of the Copy Number. Plant J. 9: 787-797.

Flavell, R.B. 1994. Inactivation of Gene Expression in Plants as A Consequence of Specific Sequence Duplication. Proc Natl Acad Sci USA 91: 3490-3496.

Hayashi, T. 1989a. Xyloglucans in the Primary Cell Wall. Annu Rev Plant Physiol Plant Mol Biol 40: 139-168.

Hayashi, T. 1989b. Measuring $\beta$-glucan Deposition in Plant Cell Walls. In: Linskens HF and Jackson JF (Eds.) Modern Methods of Plant Analysis, Plant Fibers, Springer-Verlag, Berlin Heidelberg. pp. 138-160.

Hayashi, T.; T. Takeda. 1994. Compositional Analysis of the Oligosaccharide Units of Xyloglucans from Suspension-cultured Poplar Cells. Biosci Biotech Biochem 58: 1707-1708.

Hayashi, T.: K. Ogawa; Y. Mitsuishi. 1994. Characterization 
of the Adsorption of Xyloglucan to Cellulose. Plant Cell Physiol 35: 1199-1205.

Palauqui, J.C.; H. Vaucheret. 1998. Transgenes are Dispensable for the RNA Degradation Step of Cosuppression. Proc Natl Acad Sci USA 95: 96759680.

Park, Y.W.; K. Baba; Y. Furuta; I. lida; K. Sameshima; M. Arai; T. Hayashi.2004. Enhancement of Growth and Cellulose Accumulation by Overexpression of Xyloglucanase in Poplar. FEBS Lett 564: 183-187.

Takeda, T.; Y. Furuta; T. Awano; K. Mizuno; T. Hayashi. 2002. Suppression and Acceleration of Cell Elongation by Integration of Xyloglucans in Pea Stem Segments. Proc Natl Acad Sci USA 99: 9055-9060.

Taniguchi, T.; Y. Ohmiya; M. Kurita; M. Tsubomura; T. Kondo; Y.W. Park; K. Baba; T. Hayashi.2008. Biosafety Assessment of Transgenic Poplars Overexpressing Xyloglucanase (AaXEG2) Prior to Field Trials. J Wood Sci 54: 408-413.

Ulian, E.C.; J.M. Magill; C.W. Magill; R.H. Smith .1996. DNA Methylation and Expression of NPT II in Transgenic Petunias and Progeny. Theor Appl Genet 92: 976-981.

Updergraff, D.M. 1969. Semimicro Determination of Cellulose in Biological Material. Anal Biochem 32: 420424.

Yamamoto, M.; T. Saito; A. Isogai; M. Kurita; T. Kondo; T. Taniguchi; R. Kaida; K. Baba; T. Hayashi.2011. Enlargement of Individual Cellulose Microfibrils in Transgenic Poplars Overexpressing Xyloglucanase. J
Wood Sci 57: 71-75.

Zabotina, O.A.; W.T.G. van de Ven; G. Freshour; G. Drakakaki; D. Cavalier; G. Mouille; M.G. Hahn; K. Keegstra; N.V. Raikhel. 2008. Arabidopsis XXT5 Gene Encodes A Putative a-1,6-xylosyltransferase that is Involved in Xyloglucan Biosynthesis. Plant J 56: 101115.

H. lizuka, R. Kaida, T. Taji, Y. Sakata, T. Hayashi (correspondence)

Department of Bioscience, Tokyo University of Agriculture Setagaya-ku, Tokyo156-8502, Japan

Tel : $\quad+81-3-5477-2760$

Fax : : +81-3-5477-2760

E-mail : t4hayash@nodai.ac.jp

T. Kaku, K. Baba

Research Institute for Sustainable Humanosphere Kyoto University, Uji, Kyoto 611-0011, Japan

T. Taniguchi, M. Kurita, K. Konagaya, K. Ishii Forest Bio-Research Center, Forestry and Forest Product Research Institute, Ibaraki 319-1301, Japan

T. Kondo

Forest Tree Breeding Center, Forestry and Forest Product Research Institute, Ibaraki 319-1301, Japan 